\title{
Separación Electrostática de una Emulsión de Glicerina en Biodiésel con Aplicación de Varios Voltajes y Distancias entre Electrodos
}

\author{
Sebastian Huangal(1), Jaime L. Cieza ${ }^{(1)}$ y Antonio Gil(2) \\ (1) Facultad de Ingeniería Química e Industrias Alimentarias, Universidad Nacional Pedro Ruíz Gallo, Lambayeque, Perú. \\ (e-mail: sebashsche@gmail.com; jaimecs60@hotmail.com) \\ (2) INAMAT-Departamento de Ciencias, Edificio de los Acebos, Universidad Pública de Navarra, Campus de Arrosadía, \\ 31006-Pamplona, España. (e-mail: andoni@unavarra.es)
}

Recibido Nov. 29, 2018; Aceptado Ene. 29, 2019; Versión final Mar. 12, 2019, Publicado Oct. 2019

\begin{abstract}
Resumen
Con el propósito de aplicar una tecnología limpia y de bajo consumo energético en la separación de emulsiones, se construyó un separador electrostático de $1,0 \mathrm{dm}^{3}$. Se utilizaron electrodos de cobre rectangulares. Para los ensayos se prepararon emulsiones de glicerina $(10 \% \mathrm{v} / \mathrm{v})$ en biodiésel. Como agente emulsificante se utilizó licitina. Los voltajes empleados fueron 3000, 6000 y 9000 voltios; y las distancias entre electrodos fueron 2, 4 y $6 \mathrm{~cm}$. Para cada ensayo se alcanzó la separación completa de la emulsión y se registraron los tiempos necesarios. Se determinó que existe un efecto tanto individual como combinado de los factores ensayados en la separación de la glicerina del biodiésel. La distancia entre electrodos fue la variable que mayor efecto produjo en la separación, seguidos del voltaje y la combinación de voltaje/distancia. Los tiempos de separación observados varían entre 8,3 y 45,3 segundos.
\end{abstract}

\section{Electrostatic Separation of a Glycerine Emulsion in Biodiesel with Application of Various Voltages and Distances between Electrodes}

\begin{abstract}
With the purpose of applying a clean and low energy consumption technology in the separation of emulsions, an electrostatic separator of $1.0 \mathrm{dm}^{3}$ was built. Rectangular copper electrodes were used. For the tests, glycerin emulsions $(10 \% \mathrm{v} / \mathrm{v})$ were prepared in biodiesel. Licitin was used as the emulsifying agent. The voltages used were 3000,6000 and 9000 volts; and the distances between electrodes were 2, 4 and $6 \mathrm{~cm}$. For each test the complete separation of the emulsion was achieved and the necessary times were recorded. It was determined that there are both individual and combined effects of the factors tested in the separation of glycerin from biodiesel. The distance between electrodes was the variable that produced the greatest effect in the separation, followed by the voltage and the voltage/distance combination. The observed separation times vary between 8.3 and 45.3 seconds.
\end{abstract}




\section{INTRODUCCIÓN}

Los recursos energéticos limitados y las regulaciones de emisiones cada vez más estrictas han motivado una intensa búsqueda de combustibles de transporte alternativos durante las últimas tres décadas (Abbaszaadeh et al., 2012). Según la directiva 2003/30/CE de la Comisión de la Unión Europea, se sugiere el uso de biocombustibles para asegurar y diversificar la oferta de energía, y a su vez, disminuir las emisiones netas de $\mathrm{CO}_{2}$ para el transporte terrestre en Europa. El biodiésel se define como los mono ésteres de alquilo de ácidos grasos obtenidos a partir de recursos renovables tales como aceites vegetales, grasas animales, aceites residuales y aceite de algas (Malhotra y Sarin, 2004; Sánchez et al, 2017). Se estima que la producción de biodiésel a nivel mundial pasará de casi 28 mil millones de litros que se producen en la actualidad, a 38,6 mil millones de litros que se tiene previsto producir en 2014 (García-Camús y García-Laborda, 2006). La producción de biodiésel a partir de abundantes bio-fuentes ha atraído la atención de las comunidades académica, así como industrial en los últimos años por su carácter renovable, biodegradable, baja emisión de gases de efecto invernadero, bajas emisiones de azufre, entre otros beneficios en comparación con los combustibles derivados del petróleo. Sin embargo, uno de los obstáculos más serios para el uso del biodiésel como combustible alternativo es el complicado y costoso proceso de purificación en su producción. Las dificultades relacionadas con la separación de la glicerina y otros reactivos y subproductos que no han reaccionado requieren el desarrollo de nuevos procesos de separación de bajo coste para este fin (Maan et al, 2010). El método de separación por gravedad no es una solución adecuada para las instalaciones de producción que buscan una configuración de flujo continuo. Tras la reacción, la glicerina se separa por decantación o centrifugación y la capa obtenida se purifica antes de usarla para sus aplicaciones tradicionales (farmacéutica, cosmética y alimentaria) o para las aplicaciones más utilizadas en los últimos años como cargas de carbono en fermentaciones, polímeros, tensoactivos, intermedios y lubricantes (Vicente et al., 2007).

El biodiésel es producido de fuentes renovables, como aceites vegetales, y puede ser mezclado en cualquier proporción con diésel mineral, procedente del petróleo, para su uso en la mayoría de motores diésel. Sin embargo, hasta ahora los fabricantes de vehículos permiten mezclas de hasta el $20 \%$ de biodiésel, llamado B20. Sólo algunos fabricantes de vehículos alemanes ofrecen motores adaptados para usarse con biodiésel al 100\%. El biodiésel es biodegradable y tiene una huella de $\mathrm{CO}_{2}$ más baja que el diésel mineral. Excepto por los NOx que se generan, las emisiones de los vehículos que usan biodiésel son menores que las correspondientes de los vehículos que utilizan gasolina. Debido a su punto de inflamación más alto, es más seguro tanto en su uso como en el almacenamiento que el diésel mineral. Sin embargo, el almacenamiento de larga duración del biodiésel u otros biocombustibles, necesita especial atención. El biodiésel es fuertemente higroscópico y puede adsorber humedad del aire, ocasionando diferentes tipos de problemas. Los hongos y las bacterias pueden causar problemas en grandes tanques de almacenamiento mientras se encuentran en presencia de agua condensada. La polimerización debido a la oxidación es otro problema común con el biodiésel. Los polímeros resultantes obstruyen los filtros de combustible con bastante rapidez. Se pueden formar vapores de metanol como resultado de la descomposición o polimerización del biodiésel y puede conducir a riesgos más altos de explosión en combinación con electricidad estática.

La producción de biodiésel a partir de aceite vegetal requiere un gran número de etapas que se detallan en la Figura 1. La separación de la glicerina del biodiésel es una de las etapas finales más importantes para obtener un biodiésel con una calidad adecuada. Los medios tradicionales para eliminar la glicerina son mediante separación por gravedad o por centrifugación (Cardeño, et al, 2011; Demirbas, 2007). Los sistemas de separación de glicerina del biodiésel por centrifugación son caros ya que su consumo de energía es elevado. En algunos casos, este método transfiere parte de la fase de biodiésel a la fase de glicerina obteniéndose una emulsión (Ganduglia et al., 2009). La separación por gravedad requiere de tiempo para separar la emulsión. Además, el biodiésel obtenido debe ser de alta calidad y que cumpla las correspondientes normativas de los países productores. En última instancia, se deben buscar otros métodos para hacer la separación fácil, rápida y menos costosa (Huangal et al. 2019). La separación mediante la aplicación de una corriente electrostática es uno de los procedimientos que se aplica en las emulsiones agua-aceite, agua-amoniaco (Mhatre et al., 2015), pero que menos información se tiene en la purificación de biodiésel. El concepto básico es que al introducir un alto voltaje a la mezcla, el líquido con una mayor conductividad se acumulará en el fondo del recipiente y el líquido con menor conductividad migrará a la parte superior. Austic y Shore (2009) llevaron a cabo una serie de experimentos para separar glicerina y biodiésel. Debido a la mínima información disponible sobre la resistencia de la glicerina o del biodiésel, comenzaron con una derivación indirecta de estas mediciones aplicando un voltaje constante y midiendo la potencia del sistema. Los resultados de este experimento indican que una combinación de alto voltaje, distancias grandes entre electrodos y bajo contenido de ácidos grasos pueden separar rápidamente la glicerina. Además hay un voltaje umbral que se puede alcanzar para maximizar el proceso. Una vez superado este umbral, no proporciona un mayor beneficio incrementar el voltaje. Aunque el análisis y viabilidad de este método parece bastante simple, las consideraciones de seguridad asociadas con altos voltajes sumergidos en líquidos deben explorarse para determinar aún más su posible aplicación y desarrollo. 
En el presente trabajo de investigación se plantea estudiar cómo afectará la aplicación de distintos voltajes y distancias entre electrodos sobre el tiempo de separación de la glicerina y el biodiésel por coagulación electrostática de la glicerina. Se propone como objetivo general determinar la variación del tiempo de separación de la glicerina del biodiésel por coagulación electrostática aplicando varios voltajes y distancias entre electrodos. Para lograr este objetivo general se formularon tres objetivos específicos: determinar las condiciones en las que se aplicarán campos eléctricos para separar la glicerina del biodiésel, fijar los voltajes y separación entre electrodos que se van a estudiar, y determinar la combinación de voltaje y separación entre electrodos más adecuada para separar la glicerina del biodiésel.

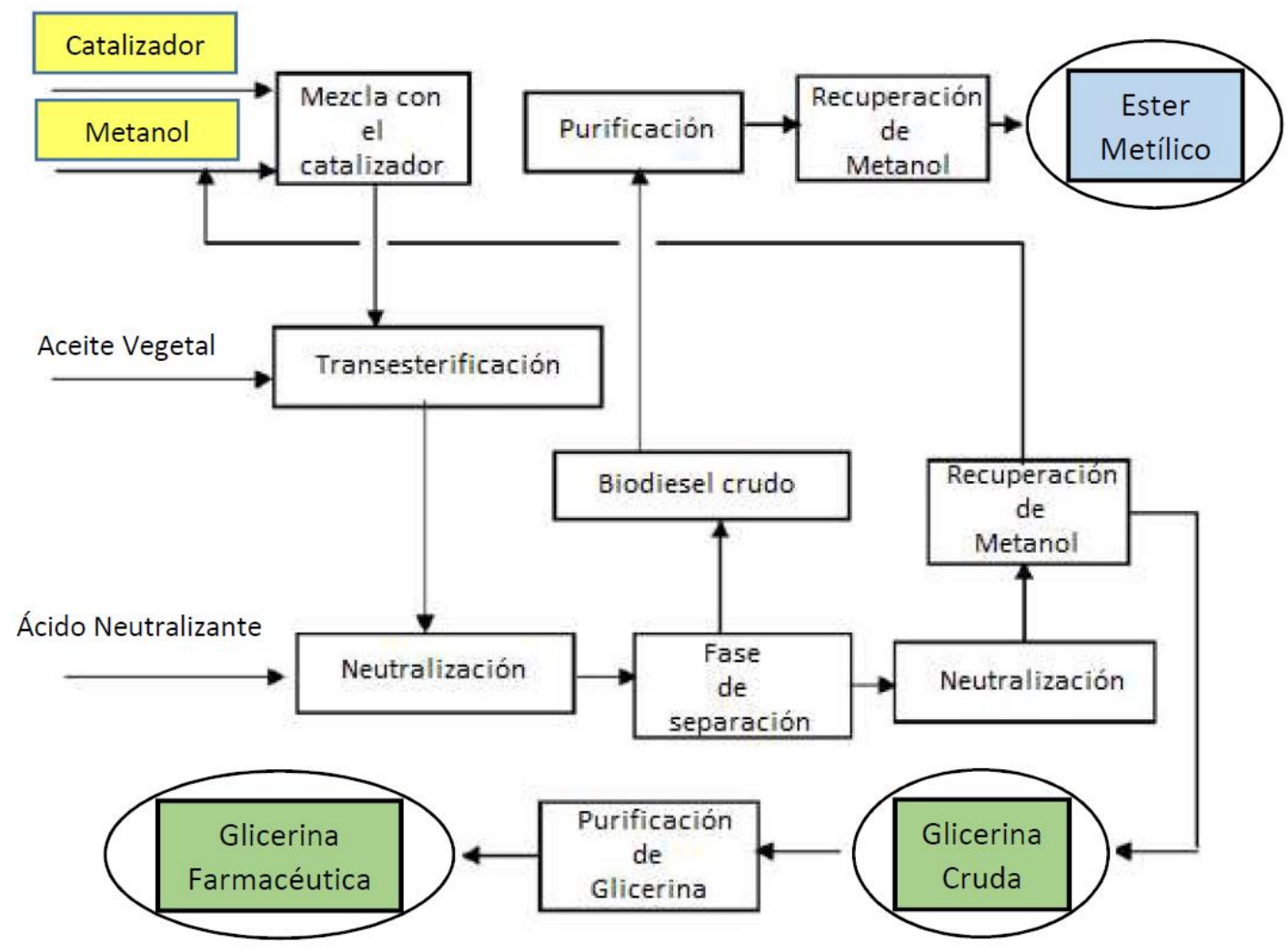

Fig. 1: Esquema tradicional de producción de biodiésel a partir de aceites vegetales.

\section{METODOLOGÍA}

Las muestras a analizar se sintetizaron con un $10 \% \mathrm{v} / \mathrm{v}$ de glicerina y $90 \%$ de biodiésel. La glicerina utilizada fue del grado comercial de "alta gravedad" que según sus especificaciones tiene una concentración superior a 99,0\% en peso de glicerina (Posada et al., 2009). El biodiésel utilizado, adquirido de la planta de producción de biodiésel de Universidad Agraria La Molina (UNALM), presentó el análisis que se muestra en la Tabla 1. En esta tabla se presenta a modo de comparación, dado que los procesos a partir de los que se obtienen pueden ser muy diferentes, los valores de la Norma USA para el biodiésel (ASTM D-6751) y de Norma Europea para el biodiésel (D6751-09). La composición utilizada se asemeja a la composición con que sale la emulsión glicerina en biodiésel del reactor de transesterificación. Se prepararon un total de $9 \mathrm{dm}^{3}$ de mezcla glicerina-biodiésel. Para ayudar a formar la emulsión se utilizó licitina en una dosis de $2 \mathrm{~g} / \mathrm{dm}^{3}$. En cada ensayo se utilizaron $1 \mathrm{dm}^{3}$ de muestra y se aplicaron los diferentes voltajes y distancias entre electrodos, con tres repeticiones para cada muestra.

Variables estudiadas: 1) distancias entre electrodos: se ensayaron tres separaciones de los electrodos de cobre: 2, 4 y $6 \mathrm{~cm}$. Las distancias se fijaron haciendo la lectura con un calibre digital; 2) voltaje aplicado: se aplicaron tres voltajes: 3000 (100), 6000 (50) y 9000 V (35 mA). Para la medición se utilizó un multímetro digital; 3) tiempo de separación: se define como la duración del proceso para la separación de la glicerina del biodiésel cuando se aplicaron las distintas combinaciones de distancias entre electrodos y voltajes.

Toma de resultados: El método utilizado para la recolección de resultados fue la observación estructurada, realizándose el registro sistemático en cuadros de comportamiento de las variables estudiadas: voltaje aplicado, distancias entre electrodos y tiempo de separación. Los datos de recolectaron de acuerdo al siguiente esquema que se incluye en la Tabla 2. 
Tabla 1: Características del biodiésel utilizado en este trabajo y comparación con las normas americanas y europeas.

\begin{tabular}{|l|c|c|c|}
\hline \multicolumn{1}{|c|}{ Parámetro } & $\begin{array}{c}\text { Biodiésel de } \\
\text { palma } \\
(\text { UNALM) }\end{array}$ & $\begin{array}{c}\text { Norma USA } \\
\text { biodiésel } \\
\text { ASTM D-6751 }\end{array}$ & $\begin{array}{c}\text { Norma EU } \\
\text { biodiésel } \\
14214-2008\end{array}$ \\
\hline Punto de inflamación ( ${ }^{\circ} \mathrm{C}$, mín) & 154 & 150 & 93 \\
\hline Agua y sedimentos (\% vol, máx) & 0,070 & 0.05 & 0,05 \\
\hline Carbón Conradson (\% masa, máx & 0,41 & 0,05 & 0,03 \\
\hline Cenizas (\% masa, máx) & 0,006 & - & 0,003 \\
\hline Temperatura de destilación (Al 90\%, $\left.{ }^{\circ} \mathrm{C}\right)$ & 343 & 360 & 350 \\
\hline Viscosidad cinemática $40^{\circ} \mathrm{C}(\mathrm{cSt})$ & 4,0 & $1,9-6,0$ & 3,5 \\
\hline Corrosión lámina de cobre (máx) & $\mathrm{N}^{\circ} 1^{\text {a }}$ & $\mathrm{N}^{\circ} 3$ & $\mathrm{~N}^{\circ} 1$ \\
\hline Número de cetano (mín) & 48,6 & 47 & 51 \\
\hline Azufre (\% masa, máx) & 0,03 & 0,05 & 0,01 \\
\hline
\end{tabular}

Tabla 2: Variables experimentales estudiadas en este trabajo.

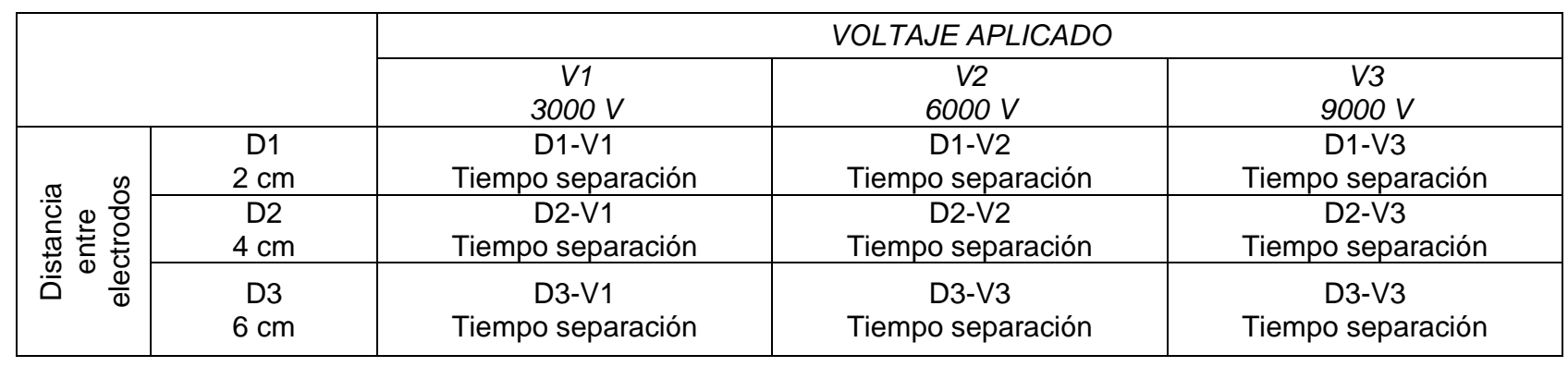

Como separador electrostático se utilizó un depósito cilíndrico de PVC transparente de 1,5 dm³ de volumen total. El separador presentaba una base de $10 \mathrm{~cm}$ de diámetro y una boca de $8 \mathrm{~cm}$ de diámetro. La altura total del cilindro fue de $18 \mathrm{~cm}$. Al depósito se adaptó una regla que permitió graduar el volumen del separador electrostático de 0 a $1 \mathrm{dm}^{3}$, con separaciones de $10 \mathrm{~cm}^{3}$. Era posible realizar el seguimiento del volumen de separación de la glicerina en todo instante. Como electrodos se utilizaron dos barras de cobre de $13 \mathrm{~cm}$ de largo, 3,5 cm de ancho y $2 \mathrm{~mm}$ de espesor. Para tener una separación fija entre los electrodos, estos se sujetaron sobre un juego de barras de plástico con graduaciones de 2, 4 y $6 \mathrm{~cm}$. Como fuente de alimentación se utilizó un trasformador de corriente eléctrica de $220 \mathrm{~V}$ que permitió regular los voltajes a 3000 (100), 6000 (50) y $9000 \mathrm{~V}$ (35 mA). La corriente empleada fue corriente alterna. Un esquema de la instalación experimental utilizada se representa en la Figura 2.

Procedimiento experimental: en primer lugar se preparó un litro de la emulsión de glicerina en biodiésel con ayuda de licitina como emulsificante. Se utilizó $10 \%$ (v/v) de glicerina y $2 \mathrm{~g}$ de licitina. Para la preparación se utilizó un agitador magnético regulado a $500 \mathrm{rpm}$ y se agitó hasta que la mezcla presentaba un aspecto lechoso (véase Figura 3). El equipo de separación electrostático se llenó con la emulsión y se conectó el transformador a la alimentación de electricidad. A continuación se registró el tiempo de separación de la emulsión, facilitado porque la glicerina tiene un aspecto más oscuro que el biodiésel.
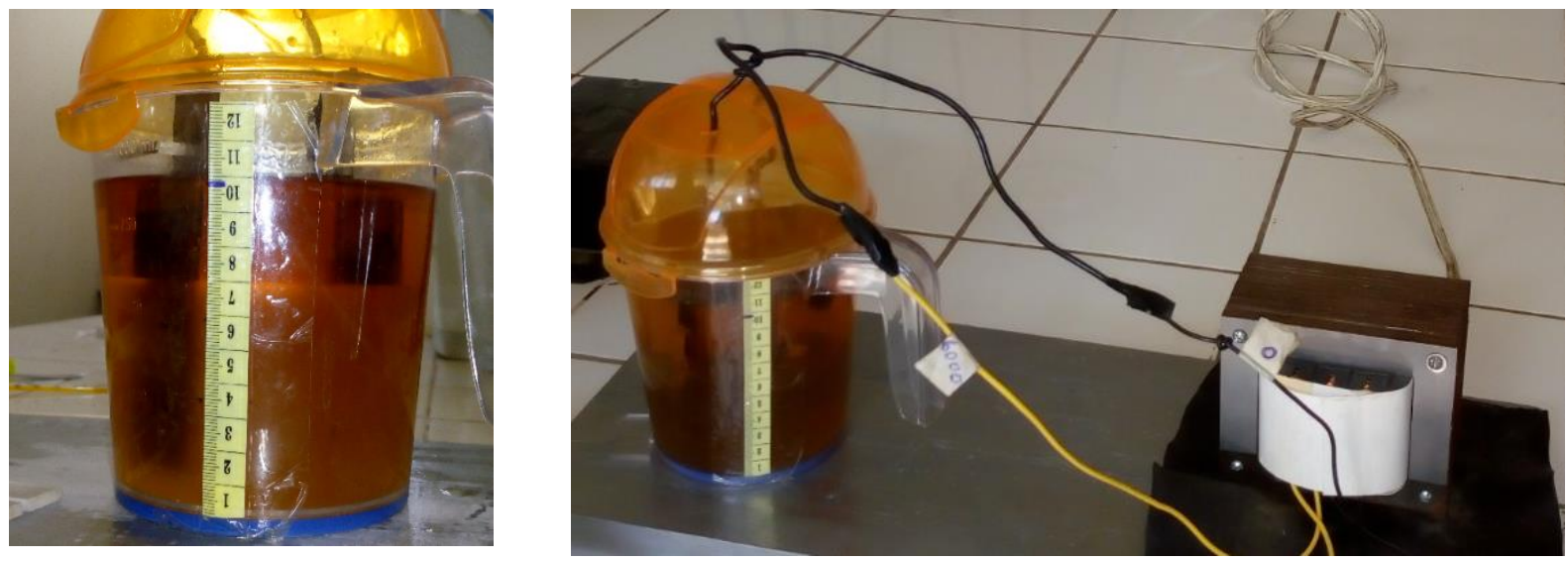

Fig. 2: Detalle del procedimiento experimental. 


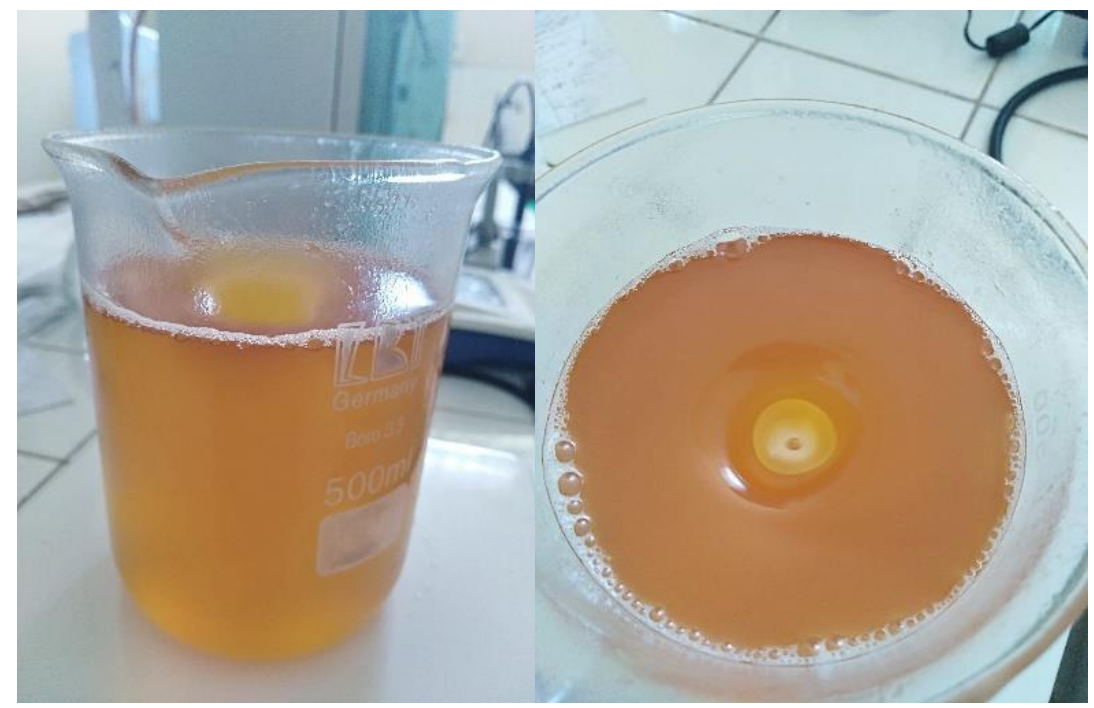

Fig. 3: Detalle de las emulsiones iniciales.

\section{RESULTADOS Y DISCUSIÓN}

De acuerdo con los datos incluidos en la Tabla 2, el biodiésel utilizado en el presente trabajo se acerca a las especificaciones que se fijan en la Norma USA, ASTM D-6751. Los promedios de tiempos alcanzados empleando la combinación de tres voltajes $(3000,6000$ y $9000 \mathrm{~V})$ y tres distancias entre electrodos $(2,4$ y 6 $\mathrm{cm}$ ) se incluyen en la Tabla 3 . De los resultados se observa que la separación más rápida entre la glicerina y el biodiésel se logra con la combinación de $9000 \mathrm{~V}$ y una separación entre electrodos de $2 \mathrm{~cm}$. El tiempo transcurrido fue de 8,3 segundos. En cambio, para un voltaje de $3000 \mathrm{~V}$ y una distancia entre electrodos de 6 $\mathrm{cm}$ se logró la misma separación en un tiempo de 45,3 segundos. En los últimos años han aparecido en la bibliografía muy pocos trabajos de varios autores que utilizan esta técnica para purificar biodiésel obtenido de fuentes diversas (Abbaszadeh et al, 2013, 2014; Barakat y Mayer-Laigle, 2017; Ratanabuntha et al, 2018; Susumu, 2018; Trisnaliani y Zikri, 2018; Utama Putra et al, 2018; Xie et al, 2011). En la mayoría de los trabajos no se da información detallada de cómo llevan a cabo los procesos. Así, Susumu et al (2018) fijan la distancia y voltaje del sistema que emplean $(1,5 \mathrm{~cm}$ y 12 voltios), sin indicar el tiempo necesario para obtener el biodiésel con la pureza que requiere la normativa de Indonesia. El tiempo máximo utilizado en los experimentos es de $30 \mathrm{~min}$. El biodiesel es sintetizado a partir de un residuo de aceite de cocina con un rendimiento del $83,3 \%$. Antes de llevar a cabo la purificación, el biodiesel es filtrado para eliminar impurezas. Utama-Putra et al (2018) observan separación entre biodiesel y glicerina transcurridos 2 min y 10 segundos tras aplicar un voltaje de 33,5 voltios, una corriente de 1,1 A y con una separación de los electrodos de 1,5 $\mathrm{cm}$. Los autores encuentran que la viscosidad y el contenido en agua del biodiesel disminuyen al aumentar el voltaje aplicado. Camara et al (2011) utilizan diferentes tipos de electrodos y obtienen una separación eficaz en un tiempo de 19 - 45 segundos, pero no indican los voltajes aplicados.

En el caso del trabajo presentado por Abbaszadeh et al (2014) proporcionan más detalles experimentales incluyendo la geometría del electrodo (placas, alambres, barras), la distancia entre los electrodos (3, 6 y 9 $\mathrm{cm})$, el voltaje y la intensidad de corriente (3400 V - $112 \mathrm{~mA}, 6800 \mathrm{~V}-56 \mathrm{~mA}, 10200 \mathrm{~V}-38 \mathrm{~mA}, 13600 \mathrm{~V}-$ $28 \mathrm{~mA}$ ). En este caso también emplean un residuo de aceite de cocina para sintetizar el biodiesel. Los autores observaron que cuando se fija la distancia entre los electrodos a $3 \mathrm{~cm}$, el tiempo requerido para separar la glicerina del biodiesel se encuentra en el rango más bajo, entre 19 a 45 segundos. El tipo de electrodo también influye, siendo mayor en el caso de los electrodos tipo alambre. Si se aumenta la distancia entre los electrodos, se requiere más tiempo para la separación. Los autores explican este resultado porque disminuye el campo electrostático y al mismo tiempo la coagulación del biodiésel. El comportamiento que se presenta en este trabajo corrobora los resultados obtenidos por Abbaszadeh et al (2012) y Noik et al. (2006) quienes indicaron que un mayor campo eléctrico tiene una influencia directa sobre el tiempo de separación de emulsiones de agua en aceite. Se debe tener en cuenta que el campo eléctrico es directamente proporcional al voltaje aplicado e inversamente proporcional a la distancia entre electrodos. Sólo a título informativo dado que los principios en los que se basa la separación son diferentes cabría resaltar que los tiempos obtenidos en la separación electrostática de la emulsión de glicerina en biodiésel son menores que los tiempos necesarios si se aplican las tecnologías tradicionales. Por ejemplo, los tiempos necesarios si se emplea un coalescedor mecánico varían entre 1 a 2 h (Santacesaria et al, 2012). Si es un sedimentador por gravedad la separación se puede alcanzar transcurrido un día (Hawash, 2016). 
Tabla 3: Tiempo promedio, medido en segundos, de separación electrostática de emulsiones de glicerina en biodiésel empleando combinación de voltajes y distancias entre electrodos.

\begin{tabular}{|l|c|c|c|c|}
\hline \multirow{2}{*}{} & & \multicolumn{3}{|c|}{ Voltaje aplicado (voltios) } \\
\cline { 2 - 5 } & & V2: 3000 V & V2:6000 V & V3: $9000 \mathrm{~V}$ \\
\hline \multirow{3}{*}{$\begin{array}{l}\text { Distancia } \\
\text { entre } \\
\text { electrodos }\end{array}$} & $\mathrm{D}_{1::} 2 \mathrm{~cm}$ & 17,0 & 13,0 & 8,3 \\
\cline { 2 - 5 } & $\mathrm{D}_{2}: 4 \mathrm{~cm}$ & 31,7 & 22,0 & 10,3 \\
\cline { 2 - 5 } & $\begin{array}{c}\mathrm{D}_{3} \\
613 \mathrm{~cm}\end{array}$ & 45,3 & 31,0 & 19,3 \\
\hline
\end{tabular}

De igual forma que con las tecnologías tradicionales y teniendo en cuenta además que las cantidades a separar serían muy diferentes, así como el coste económico, la tecnología que más se podría acercar en tiempo de separación sería la centrifugación, que necesita sólo unos minutos para conseguirlo. Sin embargo, el consumo de energía eléctrica elevaría los costos de producción. Por ejemplo, según el catálogo de un fabricante de centrifugas se necesita un motor de $22 \mathrm{~kW}$ para procesar de 5 a 17 toneladas por día de biodiésel (Alfa Laval, 2014). Como alternativa tecnológica más eficaz se tendrían los procesos en los que se emplean líquidos iónicos (Ho y col., 2015). Estos procesos requieren segundos para separar la emulsión de glicerina en biodiésel. El inconveniente es la contaminación del producto principal (el biodiésel) y el subproducto (glicerina) dado que presentarán restos de líquidos iónicos. La tecnología empleada en la presente investigación se considera una tecnología limpia debido a que no se usa otros reactivos y no se produce tampoco otros componentes, asegurando que el biodiésel y glicerina obtenidos sean de alta pureza

El análisis factorial de la varianza del tiempo de separación establecido por las combinaciones de los valores de las variables voltaje y distancia se incluye en la Tabla 4. El software utilizado fue SPSS-IBM. A partir de los valores de significancia obtenidos menores de 0,05 , se puede asegurar con una confianza del $95 \%$ que las variables ensayadas, voltaje y distancia entre electrodos, afectan al tiempo de separación de las emulsiones de glicerina en biodiésel. A partir de los valores $\mathrm{F}$ obtenidos, el factor que más efecto tiene de forma individual es la distancia entre electrodos $(F=543,146)$, aunque no se aleja mucho del efecto que presenta el voltaje aplicado $(F=516,293)$. Estos resultados indican que el tiempo de separación promedio difiere significativamente de acuerdo al voltaje utilizado, así como también de la distancia entre los electrodos. Si se analiza el valor $F$ combinado Voltaje $x$ Distacia $(F=40,683)$, que permite establecer la interacción de ambos factores en el tiempo de separación, este coeficiente es mucho menor que los valores individuales, resultado que permite deducir que el efecto combinado de los dos factores ensayados es menor que los factores individuales. Los resultados de estos efectos se incluyen en la Fig.4.

Al representar los tiempos promedios obtenidos frente al voltaje aplicado para cada distancia entre electrodos, se observa que las pendientes son mayores, y parecidas entre si, cuando las distancias entre los electrodos son de 4 y $6 \mathrm{~cm}$ (véase Figura 4, izquierda). Sin embargo, cuando la distancia es de $2 \mathrm{~cm}$, la pendiente observada es menor. Este resultado podría interpretarse como un menor efecto del voltaje aplicado. Por otro lado, para el voltaje de $9000 \mathrm{~V}$, los tiempos necesarios para lograr la separación de la emulsión se aproximan entre si, lo que indicaría que se puede ajustar el voltaje a valores menores de $9000 \mathrm{~V}$ con objeto de ahorrar energía. De igual forma, al representar los tiempos promedios obtenidos frente a la distancia entre electrodos para cada voltaje aplicado, se observa que la distancia entre electrodos tiene un mayor efecto cuando se aplican 9000 V (véase Figura 4, derecha). Esta conclusión se puede deducir de los comportamientos observados, si es creciente o decreciente, como es el caso de los resultados presentados en la Figura 4, izquierda. A medida que aumenta el voltaje la tendencia es decreciente, con un comportamiento ascendente si se tiene en cuenta la distancia entre los electrodos.

La tecnología empleada en este trabajo se puede considerar limpia debido a que no se utilizan reactivos químicos, y además se consume una cantidad de energía muy baja. Así, el consumo de energía calculado para un litro de disolución separada en un tiempo de 8,3 segundos es de aproximadamente $7,3 \cdot 10^{-4} \mathrm{~kW}$-h. Para un volumen de $200 \mathrm{dm}^{3}$, capacidad de una centrifuga, el consumo sería 0,146 kW-h. En cambio, si se aplicase la centrifugación para tratar $200 \mathrm{dm}^{3}$, con una potencia instalada de $20 \mathrm{~kW}$, el consumo sería de 0,67 kW-h, un valor de casi 4,5 veces mayor que la energía que se consumiría si se utilizase un separador electrostático. Se recomienda la aplicación de la separación electrostática como tecnología limpia en la etapa de separación de la emulsión de glicerina en biodiesel. Ensayar con emulsiones reales, para medir el efecto sobre el tiempo de separación de otras sustancias presentes en una emulsión. Realizar una evaluación económica detallada del beneficio de esta tecnología limpia, en comparación con las que se aplican actualmente, para ser usada a nivel industrial. 
Tabla 4: Análisis factorial de varianza del tiempo de separación electrostática aplicando diferentes voltajes y distancias entre electrodos.

\begin{tabular}{|c|c|c|c|c|c|}
\hline Origen & $\begin{array}{c}\text { Suma de } \\
\text { cuadrados tipo II }\end{array}$ & $\begin{array}{c}\text { Grados } \\
\text { de } \\
\text { libertad }\end{array}$ & $\begin{array}{c}\text { Media } \\
\text { cuadrática }\end{array}$ & $\begin{array}{c}\text { Razón de } \\
\text { Fisher }\end{array}$ & $\begin{array}{c}\text { Nivel de } \\
\text { sinificación }\end{array}$ \\
\hline Modelo corregido & 3464,667 & 8 & 433,083 & 285,201 &, 000 \\
\hline Intersección & 13068,000 & 1 & 13068,000 & 8605,756 &, 000 \\
\hline Voltaje & 1568,000 & 2 & 784,000 & 516,293 &, 000 \\
\hline Distancia & 1649,556 & 2 & 824,778 & 543,146 &, 000 \\
\hline Voltaje x Distancia & 247,111 & 4 & 61,778 & 40,683 &, 000 \\
\hline Error & 27,333 & 18 & 1,519 & & \\
\hline Total & 16560,000 & 27 & & & \\
\hline
\end{tabular}
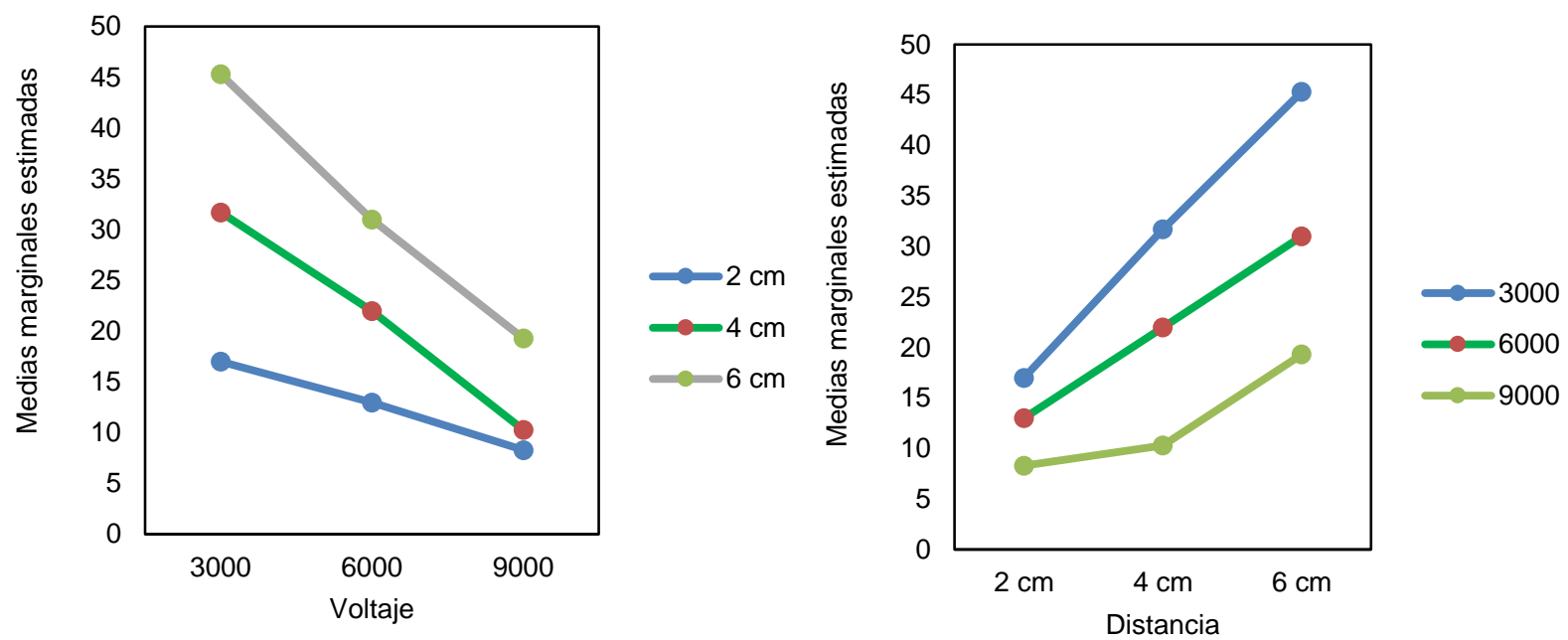

Fig. 4: Efecto individual del voltaje aplicado (izquierda) y de la distancia entre electrodos (derecha) durante la separación electrostática de una emulsión de glicerina en biodiésel.

\section{CONCLUSIONES}

En este trabajo se estableció que existe efecto individual y combinado del voltaje y la distancia entre electrodos sobre el tiempo de separación electrostática de una emulsión de glicerina en biodiésel. El mayor efecto lo ejerció la distancia entre electrodos, seguido del voltaje, y menos efecto tuvo la combinación de voltaje y distancia entre los electrodos. Se evaluó la combinación factorial de tres voltajes (3000, 6000 y $9000 \mathrm{~V})$ y tres distancias entre electrodos (2, 4 y $6 \mathrm{~cm}$ ), obteniendo para todos tiempos de separación aceptables desde 8,3 hasta 45,3 segundos, y con bajo consumo de energía.

\section{AGRADECIMIENTOS}

S. Huangal desea agradecer a la Unión Europea por la subvención económica, a través del programa Erasmus+, que permite llevar a cabo parte de este trabajo de investigación. A. Gil también desea agradecer al Banco Santander por la financiación económica a través del Programa de la Intensificación de la Investigación.

\section{REFERENCIAS}

Abbaszaadeh, A., B. Ghobadian y otros dos autores, Current biodiesel production technologies: A comparative review, doi: 10.1016/j.enconman.2012.02.027, Energy Conver. Management63, 138-148 (2012)

Abbaszaadeh, A., B. Ghobadian y otros dos autores, Electrostatic coagulation for separation of crude glycerin from biodiesel, Adv. Environ. Biol., 8, 321-324 (2014)

Abbaszaadeh, A., G. Najafi y A. Ghohadian, Design, fabrication and evaluation of a novel biodiesel processor system, J. Renov. Energy Technol. Res., 2, 249-255 (2013)

Alfa Laval, BD 55. Separation system for biodiesel processing, Catalogue PCHS00001EN 0603 (2014) 
Austic, G. y S. Shore, Characterization of the effect of high voltage current on the enhancement of biodiesel/glycerin separation, North Carolina State Univ. North Carolina (2010)

Barakat, A. y C. Mayer-Laigle, Electrostatic separation as an entry into environmentally eco-friendly dry biorefining of plant materiales, doi: 10.4172/2157-7048.1000354, J. Chem. Eng. Process, 8, 1000354 (2017)

Camara, L.T.D. y D.A.G. Arnda, Reaction kinetics study of biodiesel production from fatty acid esterification with ethanol, doi: 10.1021/ie1005806, Ind. Eng. Chem. Res., 50, 2544-2547 (2011)

Cardeño, F., L.J. Gallego y L.A. Ríos, Refinación de la fase glicerina del biodiesel de aceite de palma empleando ácidos minerales, doi: 10.4067/S0718-07642011000600003, Inf. Tecnol., 22, 15-24 (2011)

Demirbas, A., Importance of biodiesel as transportation fuel, doi: 10.1016/j.enpol.2007.04.003, Energy Policy, 35, 46614670 (2007)

Ganduglia, F., J.G. León y otros cinco autores, Manual de Biocombustibles. Asociación Regional de Empresas de Petróleo y Gas Natural de América latina y el Caribe (2009)

García-Camús, J.M. y J.A. García Laborda, Informe de vigilancia tecnológica, Biocarburantes líquidos: biodiésel y bioetanol, Fundación para el conocimiento madri+d CEIM (2006)

Hawash, S., N. Attia y otros dos autores, Quality improvement of produced biodiesel and glycerol from jatropha oil transesterification, Int. J. Sci. \& Eng. Res., 7, 375-380 (2016)

Ho, K.C., K. Shahbaz y otros cuatro autores, Removal of glycerol from palm oil-based biodiesel using new ionic liquids analogues, J. Eng. Sci. Technol. EURECA Special Issue, 98-111 (2015)

Huangal S., J.L. Cieza y A. Gil, Tecnologías eficientes de purificación del biodiésel crudo, Industria Química, febrero, 76 81 (2019)

Maan, H., S.M. Farouq y otros dos autores, A novel technique for separating glycerine from palm oil-based biodiesel using ionic liquids, doi: 10.1016/j.fuproc.2009.09.002, Fuel Proces. Technol., 91, 116-120 (2010)

Malhotra, R.K. y R. Sarin, Bio-diesel for energy security, environment protection and employment generation, SAE Publication, 2004-28-030 (2004)

Mhatre, S., V. Vivacqua y otros siete autores, Electrostatic phase separation: A review, doi: 10.1016/j.cherd.2015.02.012, Chem. Eng. Res. Des., 96, 177-195 (2015)

Noik, C., J. Chen y C.S.H. Dalmazzone, Electrostatic demulsification on crude oil: a state-of-the-art review, doi: 10.2118/103808-MS, Soc. Pet. Eng., SPE-103808-PP, 1-12 (2006)

Posada, J., C. Cardona y dos autores más, Capítulo 6: Bioglicerol como materia prima para la obtención de productos de valor agregado; en Avances Investigativos de la Producción de Biocombustibles, 1aㅡ Ed., Gráficas Tizán, Colombia (2009)

Ratanabuntha, Th., K. Tonmitr y A. Suksri, Acceleration in biodiesel production from palm oil process by high voltaje electric field, doi: 10.12720/sgce.7.3.225-230, Int. J. Smart Grid Clean Energy, 7, 225-230 (2018)

Sánchez, L., B.A. Llano y L.A. Rios, Producción de diésel renovable a partir de aceite de higuerilla mediante catalizadores de níquel-molibdeno soportados sobre alúmina, doi: 10.4067/S0718-07642017000100003, Inf. Tecnol., 28, 13-24 (2017)

Santacesaria, E, G. Vicente y otros dos autores, Main technologies in biodiesel production: State of the art and future challenges, doi: 10.1016/j.cattod.2012.04.057, Catal. Today, 195, 2-13 (2012)

Susumu, R. y S. Yusi, Biodiesel production from waste cooking oil using electrostatic method, doi: 1024845/ijfac.v3.i3.71, J. Fundam. Appl. Chem., 3, 71-76 (2018)

Trisnaliani, L. y A. Zikri, Separation of glycerol from biodiesel oil products using high voltage electrolysis method, doi: 10.24845/ijfac.v3.i1.07, J. Fundam. Appl. Chem., 3, 7-11 (2018)

Utama Putra, J.L. Kalsum e Y. Bow, Effect of DC voltaje on prototype of biodiesel electrostatic separator with glycerin from waste cooking oil, doi: 1024845/ijfac.v3.i3.89, J. Fundam. Appl. Chem., 3, 89-93 (2018)

Vicente, G., M. Martínez y J. Aracil, Optimisation of integrated biodiesel production. Part I. A study of the biodiesel purity and yield, doi: 10.1016/j.biortech.2006.07.024, Bioresource Technol., 98, 1724-1733 (2007)

Xie, Q.-G., W. Taweerpreda y otros dos autores, Separation of oily sludge and glycerol from biodiesel processing waste by coagulation, Songklanakarin J. Sci. Technol., 33, 699-703 (2011) 